\title{
Changes in depth distribution and biomass of sublittoral seaweeds at Helgoland (North Sea) between 1970 and 2005
}

\author{
Constanze Pehlke ${ }^{1,2, *}$, Inka Bartsch ${ }^{1}$ \\ ${ }^{1}$ Alfred Wegener Institute for Polar and Marine Research, Am Handelshafen 12, 27570 Bremerhaven, Germany \\ ${ }^{2}$ Present address: University of Rostock, Albert Einstein Str. 3, 18051 Rostock, Germany
}

\begin{abstract}
Recent investigations of the intertidal macrophyto- and zoobenthos of the island of Helgoland (North Sea) revealed that species composition and spatial extent of communities have changed within the last century. To evaluate the situation in the subtidal, a diving study from the late 1960s was repeated with comparable methods in 2005 and 2006. Along 2 vertical transects, the cover of dominant brown seaweeds, Fucus serratus, Laminaria digitata, L. hyperborea, Saccharina latissima (= Laminaria saccharina), Sargassum muticum and Desmarestia aculeata, was semi-quantitatively assessed to define vegetation zones. Within each zone, all macroalgal species were estimated quantitatively in 3 to 6 random $1 \mathrm{~m}^{2}$ quadrats. Additionally, a replicated biomass survey was performed at 6 depths $(0.5,2$, 4, 6, 8 and 10 m below mean low water spring tide [MLWS]). Comparison of recent and historical data showed some characteristic changes. The previously dominant brown seaweed $S$. latissima showed a decline in the northern part of the island, but is still present at other sites. $S$. muticum invaded the kelp forest, but is not dominant within this vegetation. The vertical distribution of $L$. hyperborea increased, and its lower depth limits as well as those of various understory seaweeds deepened by about 2 to $8 \mathrm{~m}$. Biomass data followed this trend. The maximum biomass of L. hyperborea shifted from $2 \mathrm{~m}$ below MLWS in 1967 to $4 \mathrm{~m}$ below MLWS in 2005. The overall downward extension of the Laminaria forest is concordant with the increase in water transparency observed around Helgoland since 1975.
\end{abstract}

KEY WORDS: Biomass $\cdot$ Change $\cdot$ Depth limit $\cdot$ Macroalgae $\cdot$ Laminaria $\cdot$ Saccharina $\cdot$ Helgoland North Sea

Resale or republication not permitted without written consent of the publisher

\section{INTRODUCTION}

Brown seaweeds of the order Laminariales (kelps) are the major structuring element of temperate to polar coastal kelp beds worldwide (Kain 1962, Lüning 1990). A change or loss of these kelp beds would drastically affect coastal ecosystems as they provide habitat and shelter for epibionts and rich invertebrate and fish fauna (Schultze et al. 1990). They also have a wave dampening effect which is important for coastal protection (Dubi \& Tørum 1995). Since the late 1980s, there have been increasing and alarming reports of changes in the quantitative patterns of kelp communities along European coastlines and elsewhere. Eutrophication, sedimentation, invasion of foreign species and the global warming trend have been implicated but unequivocal evidence is missing (Givernaud et al. 1991, Cosson 1999, Moy et al. 2003). In the Baltic, where the ecological role of dominant kelps is taken by species of the genus Fucus, several studies revealed a decrease in the depth distribution of Fucus vesiculosus and of algal depth limits in general. This was regarded as an indirect consequence of eutrophication due to reduced light availability (Kautsky et al. 1986, Pedersen \& Snoeijs 2001).

The seaweed vegetation of Helgoland (North Sea) has been intensively studied during the past century. The investigations focused on the intertidal and were mostly descriptive or qualitative (Bartsch \& Kuhlenkamp 2000 and citations therein). Quantitative 
investigations of the intertidal marine flora at Helgoland are few (den Hartog 1959, Markham \& Munda 1980, Munda \& Markham 1982, Reichert et al. 2008). The first, and so far only, quantitative sublittoral study was conducted by Lüning, who described sublittoral algal zonation and depth limits (Lüning 1970) and biomass as well as morphometric parameters such as stipe length and leaf area index of major kelp species (Lüning 1969). More than 25 years later, de Kluijver (1997) added information on sublittoral faunistic communities, partly extending knowledge of algal depth distribution at Helgoland.

Although regular monitoring of benthic flora and fauna was not conducted on Helgoland before 2000, changes in abundances and recent species immigrations have been documented (e.g. Bartsch \& Tittley 2004, Franke \& Gutow 2004, Reichert \& Buchholz 2006). Detailed analysis of abiotic factors at Helgo-

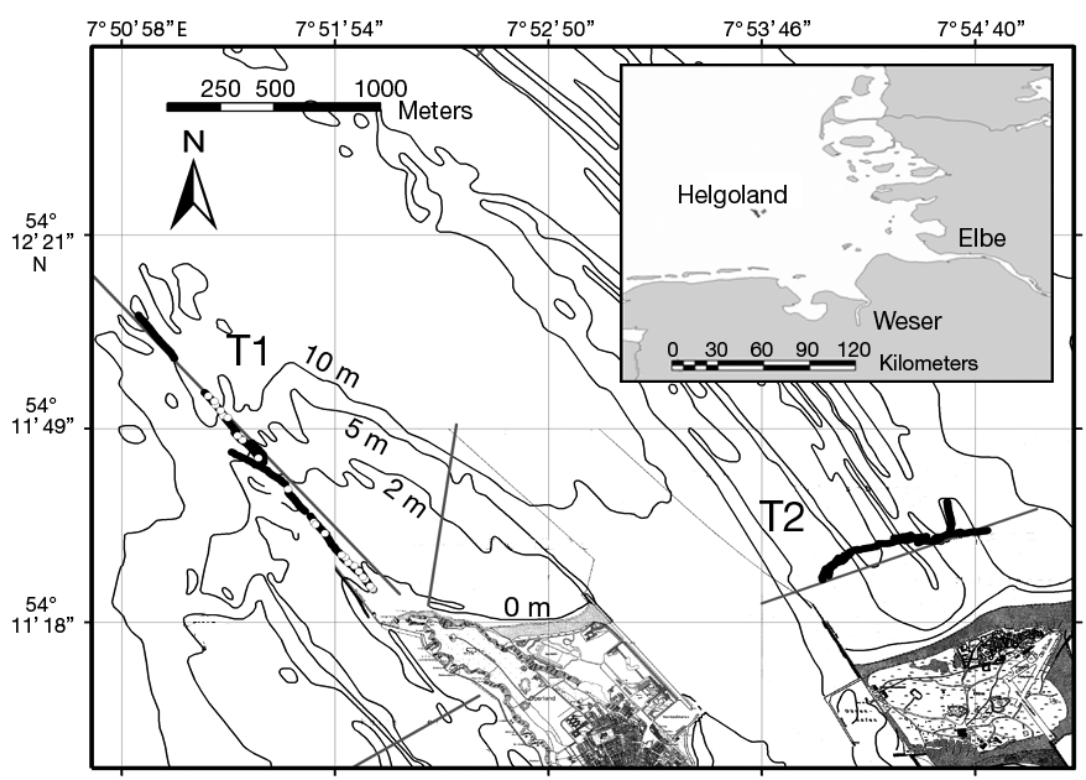

Fig. 1. Transect T1 (2005) in the north of the main island of Helgoland and Transect T2 (2006) in the north of the island Dune. Black lines: adjacent locations of $5 \mathrm{~m}$ intervals; white dots: locations of quadrats; straight lines: transects of Lüning (1970). Depth contour lines indicate 0,2, 5 and $10 \mathrm{~m}$ below mean low water spring tide (MLWS)

land (1962-2001) taken every working day revealed 3 major trends: (1) mean sea surface water temperature increased significantly by 1.13 to $1.33^{\circ} \mathrm{C}$, (2) phosphate concentration decreased by $>0.3 \mu \mathrm{mol}^{-1}$ and (3) Secchi depth increased by $1 \mathrm{~m}$ between 1975 and 2001 (Wiltshire \& Manly 2004, Wiltshire et al. 2008). Mean yearly salinity between 1975 and 2001 varied between $\sim 31$ and $\sim 33$ PSU, but without a significant trend (Wiltshire et al. 2008). Unpublished data show an increase in storminess since 1989 and a change in storm direction from predominantly W-SW to additional dominance of S-SW and E-SE (I. Bartsch pers. comm.). Furthermore, there were indications that Saccharina latissima (formerly Laminaria saccharina) stands are being lost and kelp zonation patterns are changing (I. Bartsch pers. obs.). To quantify anticipated changes in algal zonation patterns, algal depth limits and kelp biomass, the diving investigations of Lüning $(1969,1970)$ were repeated in 2005 and 2006.

\section{MATERIALS AND METHODS}

Methods used were adapted from the investigations of Lüning $(1969,1970)$ to make the datasets comparable.

\subsection{Investigation area and diving procedure}

Two sublittoral transects that had been investigated 40 years earlier by Lüning (1970) were traversed again in this study (Fig. 1). Transect T1 in the North of Helgoland has a total length of $\sim 1580 \mathrm{~m}$ and represents Transect P3 of Lüning (1970). It follows the depth gradient along the red sandstone platform of the main island for $1300 \mathrm{~m}$ in a northwesterly direction (bearing $320^{\circ}$ ). As the transect becomes much deeper after $1300 \mathrm{~m}$, carrying predominantly faunistic communities which were not in the scope of the investigation, the transect was interrupted for $\sim 220 \mathrm{~m}$. It was then continued for $\sim 280$ m over chalk cliffs, after which depths increase again and support predominantly algal communities (diving profile: Fig. 9 in Lüning 1970). Transect T2 in the north of Dune Island has a total length of $845 \mathrm{~m}$ and represents Transect P1 of Lüning (1970). It crosses the chalk and limestone cliffs, perpendicular to their depth gradients, in an east-northeasterly direction (bearing $80^{\circ}$ ).

As no coordinates were available for the P1 and P3 transects of Lüning (1970), their location was calculated as follows: Fig. 1 in Lüning (1970) was scanned (Mustek Scan Express 6000P, Mustek Optic-Computer $\&$ Communication International) and geo-referenced point by point with the help of an orthophoto of Helgoland (Land Surveying Office, Schleswig-Holstein, Germany) and the GIS Software ArcGIS 9.1 (ESRI). The start and end coordinates were determined and used as reference points for Transects T1 and T2 of the present investigation.

Fifty-one dives were carried out (T1: April 21 to August 22, 2005; T2: June 8 to July 31, 2006). Under- 
water bearings were taken with a compass (Seemann Sub). For depth determination, a digital depth gauge was used (Seemann Sub; precision: $40 \mathrm{~cm}$ ). The exact local time was logged simultaneously. The measured depth was corrected with tide level records of the tide gauge 'Helgoland Südhafen' (Water and Shipping Authority, Tönning, Germany) as had been done by Lüning (1970). To locate the present transects, the diver signaled the diving boat by tugging a cord connecting him and a surface marker buoy every 25 to $35 \mathrm{~m}$. The diving boat approached the buoy as close as possible and took a GPS reading (Magellan GPS 320, Magellan Corporation; precision: $25 \mathrm{~m}$ ). These readings were used to locate transects on a digital map (ArcGIS 9.1). Intermediate points without GPS readings were calculated in ArcInfo 9.1.

\subsection{Estimation of dominant brown seaweeds and definition of vegetation zones}

Presence/absence and percentage cover classes of the dominant brown seaweeds Desmarestia aculeata (only transect T2), Fucus serratus, Sargassum muticum, Laminaria digitata, L. hyperborea and Saccharina latissima were noted along a 3 to $5 \mathrm{~m}$ broad corridor (H. Kautsky pers. comm.) in increments of $5 \mathrm{~m}$ along T1 and T2. L. digitata and L. hyperborea were differentiated by their stipe morphology: adult L. digitata have flexible, flattened and smooth stipes with no or just few epiphytes whereas L. hyperborea have stiff, round and rough stipes with many epiphytes. L. digitata thalli lie near the substrate while L. hyperborea thalli are upright. Although these characteristics are distinct, misidentification in a few cases cannot be discounted. Cover estimation of the dominant brown seaweeds followed a modified semi-quantitative scale (Braun-Blanquet 1951, modified) with 6 cover classes (Table 1). At the beginning of each $5 \mathrm{~m}$ interval, para-

Table 1. Semi-quantitative cover classes (Braun-Blanquet 1951, modified)

\begin{tabular}{|c|c|c|}
\hline Class & $\%$ Cover & Description \\
\hline 0 & 0 & Not present \\
\hline 5 & $1-10$ & $\begin{array}{l}\text { One or few individuals present with } \\
\text { sparse ground coverage }\end{array}$ \\
\hline 25 & $11-40$ & $\begin{array}{l}\text { Clearly }<50 \% \text { of ground covered, } \\
\text { but species common }\end{array}$ \\
\hline 50 & $41-60$ & Coverage $\sim 50 \%$ \\
\hline 75 & $61-90$ & Cover clearly $>50 \%$, but $<100 \%$ \\
\hline 100 & $91-100$ & $\begin{array}{l}\text { Dense coverage, only few and small } \\
\text { gaps in canopy present }\end{array}$ \\
\hline
\end{tabular}

meters such as depth, time, substratum (red sandstone, shell limestone, chalk) and topography (solid rock, boulders, stones, gravel and shell) were recorded in addition to presence/absence and cover data. The total number of $5 \mathrm{~m}$ intervals investigated along T1 and T2 were 272 and 235, respectively.

From the data obtained, vegetation zones were defined. As Lüning (1970) did not define his 'pure' and 'mixed' vegetation stands precisely, we used the following criteria: (1) 'pure' vegetation stands, named after the dominant brown seaweed species, are those dominated by just one brown algal species with cover of 41 to $100 \%$, or with cover $<41 \%$ but without other co-occurring brown seaweeds; (2) 'mixed' stands or transition zones, named after the co-occurring dominant brown seaweeds, are comprised of 2 brown algal species in variable abundances between 11 and $90 \%$; and (3) 'red algal zones' have only foliose and filamentous red algae present. In total, 9 zones were defined (Table 2).

\subsection{Percentage cover of macrophytes}

In each vegetation zone, the percentage cover and 'rooted' local frequency (cf. Greig-Smith 1983) of all noncrustose macrophytes and all epiphytes in 3 random samples ( 6 in pure Laminaria hyperborea stand; see Table 2) were quantified with the help of a $1 \mathrm{~m}^{2}$ quadrat with 16 subdivisions. Each zone was divided into $5 \mathrm{~m}$ intervals (based on the diving segments) and numbered using GIS. Random sites were chosen by picking random numbers from these intervals. Their

Table 2. Vegetation zones of Transects T1 and T2 adopted from Lüning (1970). LAI: leaf area index; ID: identification letter for the respective zone, see Fig. 4

\begin{tabular}{|llcc|}
\hline Zone & Description & Transect & ID \\
\hline Fser & Pure Fucus serratus stands & T1 & A \\
Fser-Ldig & $\begin{array}{l}\text { Mixed F. serratus and } \\
\text { Laminaria digitata stands }\end{array}$ & T1 & B \\
Ldig & Pure L. digitata stands & T1, T2 & C \\
Ldig-Lhyp & $\begin{array}{l}\text { Mixed L. digitata and } \\
\text { L. hyperborea stands }\end{array}$ & T1, T2 & D \\
Lhyp & $\begin{array}{l}\text { Pure L. hyperborea stands } \\
\text { Lhyp-Slat }\end{array}$ & T1, T2 & E \\
& $\begin{array}{l}\text { Saccharina latissima stands } \\
\text { Slat }\end{array}$ & Pure S. latissima stands & - \\
Lhyp-park & $\begin{array}{l}\text { Pure L. hyperborea stand } \\
\text { with LAI } \leq 1\end{array}$ & T2 & - \\
Red algae & $\begin{array}{l}\text { Red algal zone without } \\
\text { dominant brown seaweeds }\end{array}$ & T1 & G \\
& & & \\
\hline
\end{tabular}


coordinates were determined and the diving boat was positioned at each of these locations in the field. At each location, a diver dived straight down and positioned the quadrat on the bottom. In each quadrat, percentage cover over ground of each species and the percentage of free substrate were estimated. Local time and depth were also noted (see above).

\subsection{Biomass survey}

All Laminaria individuals were quantitatively harvested from 3 random $1 \mathrm{~m}^{2}$ quadrats at each of 6 depths $(0.5,2,4,6,8$ and $10 \mathrm{~m}$ below mean low water spring tide [MLWS]) along T1. All other foliose and filamentous algae within a $0.25 \mathrm{~m}^{2}$ subquadrat (central 4 of 16 subdivisions) of each $1 \mathrm{~m}^{2}$ quadrat were harvested. To locate the random quadrats at each depth, a $20 \mathrm{~m}$ rope was placed perpendicular to the transect. The rope was numbered at every meter and the 3 collecting sites were randomly chosen beforehand. Fresh weights (FW) of species were determined with a Sartorius 1574 MP 8-2 (Data Weighing Systems; precision: $\pm 0.01 \mathrm{~g}$ ) after removal of epiphytes and shaking off excess water twice. Dry weights (DW) were determined after drying individual specimens at $100^{\circ} \mathrm{C}$ for $24 \mathrm{~h}$. With longer drying time, no further significant weight loss was attained. Stipe lengths of Laminaria were measured before drying. The leaf area index (LAI) was determined by taking size-calibrated digital photographs (Olympus C-8080 WZ, Olympus) of each Laminaria blade (FW > $100 \mathrm{~g}$ ). The blade area was then calculated with an image analysis program (WinFOLIA 2001a, Régent Instruments). the vegetation zones was investigated by nonparametric multidimensional scaling ( $\mathrm{n}-\mathrm{MDS}_{\text {; }}$ Bray-Curtis similarity index, square-root transformation of percentage cover data; Primer 5.1).

\section{RESULTS}

\subsection{Vertical distribution of dominant brown seaweeds}

The relative presence (RP) of Laminaria hyperborea, L. digitata, Saccharina latissima, Desmarestia aculeata (only in T2), Fucus serratus and Sargassum muticum in different cover classes (\%) normalised to all investigated $5 \mathrm{~m}$ intervals per transect is shown in Table 3. L. hyperborea dominated $\mathrm{T} 1$ and $\mathrm{T} 2$ with respective RPs of 77 and $71 \%$ followed by L. digitata with 31 and $16 \%$. In T1, S. latissima only occurred in $12 \%$ of the $5 \mathrm{~m}$ intervals, with low densities (1 to $10 \%$; cover class 5) and young thalli. In T2, it was present in $69 \%$ of the $5 \mathrm{~m}$ intervals with percentage cover (PC) values mostly between 1 to $40 \%$ (cover classes 5 and 25) and occasionally up to 91 to $100 \%$ (cover class 100). D. aculeata, which was only activly searched for in T2, occurred in nearly half of the investigated $5 \mathrm{~m}$ intervals, mostly with low coverages but sometimes with PC of up to 91 to $100 \%$. F. serratus was mainly present in the middle and lower eulittoral zone of T1 with low PC of 1 to $10 \%$. S. muticum was only present in T1 in $5 \%$ of the intervals, also with low PC of 1 to $10 \%$.

The vertical distribution of vegetation zones along both transects is shown in Fig. 2. Pure stands of Fucus serratus, Saccharina latissima, Laminaria digitata and

\subsection{Data analysis}

Quantitative data were tested for homogeneity of variance (Cochran's $C$-test). ANOVA was performed on homogeneous data or a Kruskal-Wallis ANOVA on heterogeneous data. Post hoc tests (Tukey's honestly significant difference [HSD] or multiple comparisons of mean ranks of all groups) were performed to discriminate differences between groups (Statistica 5.0, 6.0 and 8.0). To compare 2005 and 1969 data (Lüning 1969), effect size was calculated (Underwood 1997). A negative or a positive value of the effect size, with a CI not crossing the $x$-axis, indicates a significant decrease or increase in the tested variable, respectively. The similarity of
Table 3. Relative presence (number of $5 \mathrm{~m}$ intervals with occurrence of respective species in different cover classes per total number of investigated $5 \mathrm{~m}$ intervals) of dominant brown seaweeds in Transects T1 and T2 (see Fig. 1). Cover classes described in Table 1. -: no data

\begin{tabular}{|c|c|c|c|c|c|c|c|}
\hline \multirow[t]{2}{*}{ Species } & \multirow[t]{2}{*}{ Transect } & \multicolumn{5}{|c|}{ Relative presence per cover class (\%) } & \multirow{2}{*}{$\begin{array}{c}\Sigma \text { Relative } \\
\text { presence }(\%)\end{array}$} \\
\hline & & 5 & 25 & 50 & 75 & 100 & \\
\hline \multirow{2}{*}{$\begin{array}{l}\text { Laminaria } \\
\text { hyperborea }\end{array}$} & T1 & 7.8 & 12.1 & 9.3 & 22.2 & 25.7 & 77.0 \\
\hline & $\mathrm{T} 2$ & 13.7 & 13.7 & 14.1 & 13.3 & 16.1 & 71.0 \\
\hline \multirow{2}{*}{$\begin{array}{l}\text { Laminaria } \\
\text { digitata }\end{array}$} & $\mathrm{T} 1$ & 4.7 & 3.1 & 6.6 & 8.1 & 8.1 & 30.6 \\
\hline & $\mathrm{T} 2$ & 5.1 & 5.1 & 3.5 & 1.2 & 1.2 & 16.1 \\
\hline \multirow{2}{*}{$\begin{array}{l}\text { Saccharina } \\
\text { latissima }\end{array}$} & $\mathrm{T} 1$ & 11.6 & 0.4 & 0 & 0 & 0 & 12.0 \\
\hline & $\mathrm{T} 2$ & 27.4 & 25.1 & 12.2 & 5.9 & 0.8 & 68.6 \\
\hline \multirow{2}{*}{$\begin{array}{c}\text { Desmarestia } \\
\text { aculeata }\end{array}$} & T1 & - & - & - & - & - & - \\
\hline & $\mathrm{T} 2$ & 21.6 & 14.5 & 6.3 & 6.3 & 0.8 & 49.4 \\
\hline \multirow{2}{*}{$\begin{array}{l}\text { Fucus } \\
\text { serratus }\end{array}$} & T1 & 5.8 & 1.6 & 1.6 & 0.4 & 0 & 9.3 \\
\hline & T2 & 0 & 0 & 0 & 0 & 0 & 0 \\
\hline \multirow{2}{*}{$\begin{array}{l}\text { Sargassum } \\
\text { muticum }\end{array}$} & $\mathrm{T} 1$ & 5.0 & 0 & 0 & 0 & 0 & 5.0 \\
\hline & $\mathrm{T} 2$ & 0 & 0 & 0 & 0 & 0 & 0.0 \\
\hline
\end{tabular}



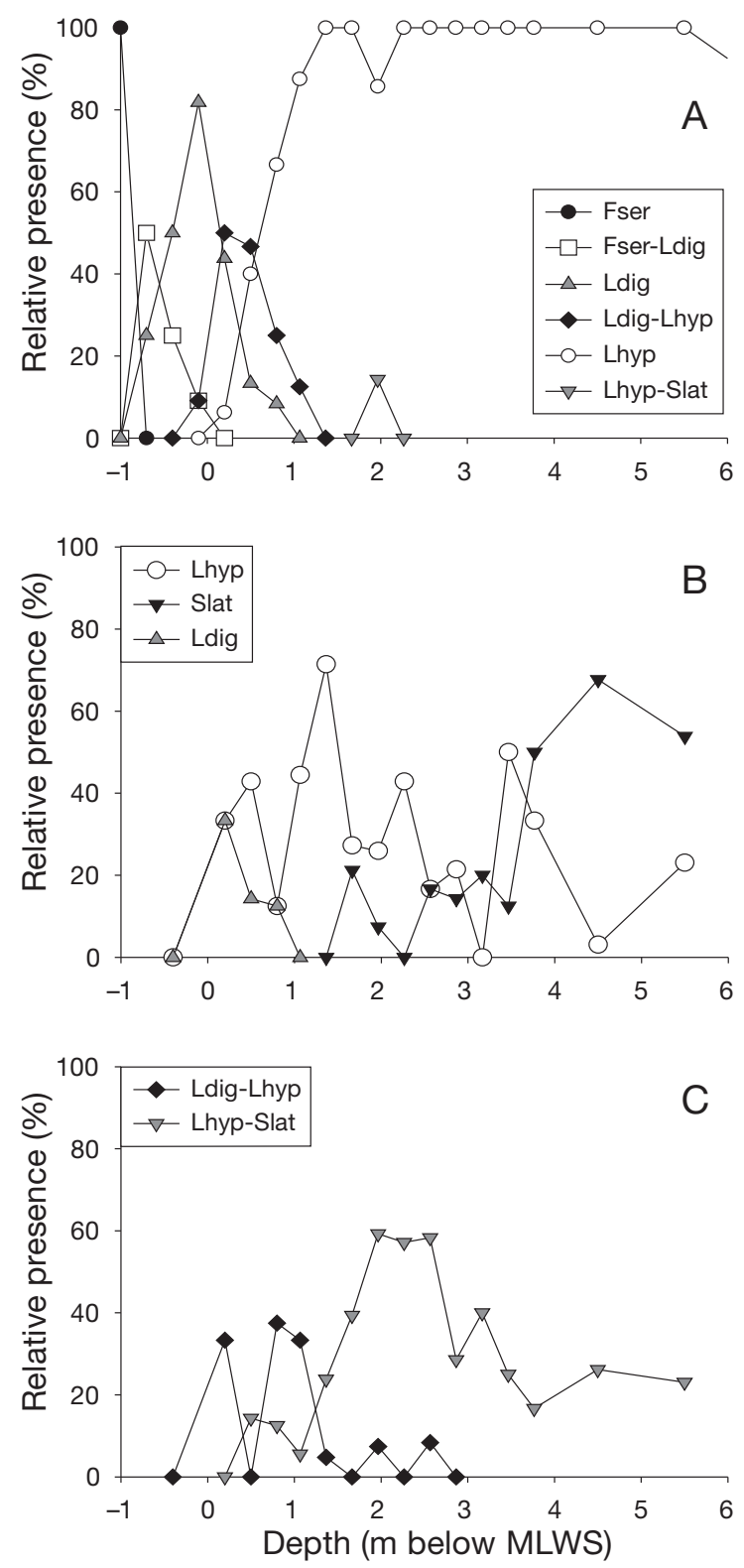

Fig. 2. Relative presence of vegetation zones dominated by single and/or mixed brown seaweeds at (A) transect T1 (2005) and $(\mathrm{B}, \mathrm{C})$ transect T2 (2006). Abbreviations of communities are explained in Table 2. For direct comparison to Lüning $(1970$; his Fig. 7) exact depth values of occurence were transformed into depth classes ( $1 \mathrm{~m}$ above-3.8 $\mathrm{m}$ below MLWS: $30 \mathrm{~cm}$ classes; 4.5-13.5 m below MLWS: $1 \mathrm{~m}$ classes). MLWS is designated as depth 0. Exact values are given in Section 3.1

L. hyperborea alternated with mixed stands. In T1 (Fig. $2 \mathrm{~A}$ ), pure $F$. serratus stands (Fser) only grew in the intertidal at $1 \mathrm{~m}$ above MLWS. Pure L. digitata stands (Ldig) were common at a depth range of $0.7 \mathrm{~m}$ above MLWS and $0.9 \mathrm{~m}$ below MLWS and pure L. hyperborea (Lhyp) between 0.2 to $10.6 \mathrm{~m}$ below MLWS (data shown only down to $6 \mathrm{~m}$ below MLWS). Among these zones were mixed stands of $F$. serratus/L. digitata (Fser-Ldig; $0.9 \mathrm{~m}$ above to $0.1 \mathrm{~m}$ below MLWS) and $L$. digitata/L. hyperborea (Ldig-Lhyp; 0 to $1 \mathrm{~m}$ below MLWS). S. latissima did not form pure stands along transect T1 and was only present at $2 \mathrm{~m}$ below MLWS in low quantities and in co-existence with $L$. hyperborea (Lhyp-Slat). Desmarestia aculeata was also present but not monitored or quantified in this transect.

Along T2 (Fig. 2B,C), Fucus serratus was absent because the entire transect was below MLWS except one point. Pure Laminaria digitata stands (Ldig) occurred between 0.3 and $0.6 \mathrm{~m}$ below MLWS, pure L. hyperborea stands (Lhyp) between 0.1 and $5.1 \mathrm{~m}$ below MLWS and pure Saccharina latissima (Slat) stands between 1.7 and $5.5 \mathrm{~m}$ below MLWS (Fig. 2B). The RP of pure $S$. latissima stands increased between 3.8 and $5.5 \mathrm{~m}$ below MLWS whereas the RP of pure L. hyperborea stands decreased at this depth range. Moreover, the RP of mixed L. hyperborea/S. latissima stands (Lhyp-Slat; Fig. 2C) at this depth range was also low. Pure stands of Desmarestia aculeata occurred between 1.5 and $5.5 \mathrm{~m}$ below MLWS, and mixed stands with Laminaria species between 1.3 and $5.4 \mathrm{~m}$ below MLWS. The RP of pure and mixed stands (maximum of 62 and $40 \%$, respectively) increased between 4 and $5.5 \mathrm{~m}$ below MLWS (not shown). Transect T2 was never deeper than $5.5 \mathrm{~m}$ below MLWS.

\subsection{Vertical distribution of all macroalgal species}

Table 4 combines presence/absence data (epiphytic and/or epilithic occurrence) along the depth gradient and depth limits observed by analysing the $1 \mathrm{~m}^{2}$ quadrats $(n=24)$ set along the vegetation zones and the 18 quadrats harvested along different depth levels for the biomass survey. Data were compared to the algal depth limits of Lüning (1970). In 2005, a total of 36 noncrustose algal species were recorded (4 Chlorophyta, 7 Phaeophyta, 25 Rhodophyta). A few species were only found as epiphytes (Ceramium deslongchampsii, Cladophora sericea, Porphyropsis coccinea) and 2 species (Cladophora sericea and Sargassum muticum) were not recorded by Lüning (1970). The number of recorded species per depth level was quite constant (17 to 21 species), except at 4 and $12 \mathrm{~m}$ below MLWS (13 species).

Eighteen out of 36 species (50\%) were recorded at depths greater by 1.9 to $8.3 \mathrm{~m}$ and 16 out of 36 species (44\%) were observed at approximately the same depth range $( \pm 1.5 \mathrm{~m})$ as those reported by Lüning (1970). One exception was Rhodochorton purpureum, a small filamentous red alga that is easily overlooked. Lüning (1970) reported $R$. purpureum at $8.0 \mathrm{~m}$ below MLWS, but it was found only at $0.4 \mathrm{~m}$ above MLWS in the pre- 
Table 4. Occurrence and lower depth limits of macroalgae at Transect T1. Comparison of recent (2005 data) and previous (1966-1968 data) depth limits is given as $\Delta \mathrm{D}$ (difference in depth distribution); -: not present; ${ }^{*}$ : no comparable data; L: epilithic, P: epiphytic on Laminaria species

\begin{tabular}{|c|c|c|c|c|c|c|c|c|c|c|}
\hline \multirow[t]{2}{*}{ Species } & \multicolumn{7}{|c|}{$\longrightarrow$ Depth (m below MLWS) - } & \multicolumn{3}{|c|}{$\begin{array}{l}\text { Lower depth limits } \\
\text { (m below MLWS) }\end{array}$} \\
\hline & 0 & 2 & 4 & 6 & 8 & 10 & 12 & $1966-1968^{a}$ & $2005^{\mathrm{b}}$ & $\Delta \mathrm{D}$ \\
\hline Ahnfeltia plicata & $\mathrm{L}$ & $\mathrm{L}$ & - & - & - & - & - & 2.7 & 2.0 & -0.7 \\
\hline Bonnemaisonia hamifera $^{\mathrm{c}}$ & - & - & - & $\mathrm{L}$ & $\mathrm{L}$ & $\mathrm{L}$ & $\mathrm{L}$ & 12.0 & 12.5 & 0.5 \\
\hline Brongniartella byssoides & - & - & $\mathrm{L}$ & $\mathrm{L}$ & $\mathrm{L}$ & $\mathrm{L}$ & $\mathrm{L}$ & 11.2 & 12.5 & 1.3 \\
\hline Bryopsis plumosa & - & - & - & - & $\mathrm{L}$ & $\mathrm{L}$ & - & 10.6 & 10.0 & -0.6 \\
\hline Ceramium deslongchampsii & - & $\mathrm{P}$ & - & - & - & - & - & 0.0 & 2.0 & 2.0 \\
\hline Ceramium virgatum & L P & $\mathrm{L}$ & $\mathrm{P}$ & $\mathrm{L}$ & $\mathrm{L}$ & - & $\mathrm{L}$ & 3.2 & 11.5 & 8.3 \\
\hline Chaetomorpha melagonium & $\mathrm{P}$ & L P & L P & - & - & $\mathrm{L}$ & - & 7.3 & 9.6 & 2.3 \\
\hline Chondrus crispus & $\mathrm{L}$ & L P & $\mathrm{L}$ & - & - & $\mathrm{L}$ & - & 2.4 & 9.6 & 7.2 \\
\hline Cladophora rupestris & $\mathrm{L}$ & - & - & - & - & - & - & 1.3 & 1.6 & 0.3 \\
\hline Cladophora sericea & $\mathrm{P}$ & $\mathrm{P}$ & - & - & - & - & - & - & 2.0 & ${ }^{*}$ \\
\hline Coccotylus truncatus & - & - & - & - & $\mathrm{L}$ & $\mathrm{L}$ & - & 7.4 & 10.0 & 2.6 \\
\hline Corallina officinalis & $\mathrm{L}$ & $\mathrm{L}$ & - & - & - & - & - & 4.7 & 2.8 & -1.9 \\
\hline Cystoclonium pupureum & - & - & - & $\mathrm{L}$ & $\mathrm{L}$ & $\mathrm{L}$ & $\mathrm{L}$ & 3.2 & 11.5 & 8.3 \\
\hline Delesseria sanguinea & $\mathrm{L}$ & L P & L P & L P & L P & $\mathrm{L}$ & $\mathrm{L}$ & 10.6 & 12.5 & 1.9 \\
\hline Desmarestia aculeata & $\mathrm{L}$ & $\mathrm{L}$ & $\mathrm{L}$ & $\mathrm{L}$ & - & $\mathrm{L}$ & $\mathrm{L}$ & 6.0 & 11.5 & 5.5 \\
\hline Desmarestia viridis & - & - & - & $\mathrm{L}$ & - & $\mathrm{L}$ & - & 7.4 & 9.3 & 1.9 \\
\hline Erythrodermis traillii & - & $\mathrm{L}$ & - & $\mathrm{L}$ & - & $\mathrm{L}$ & $\mathrm{L}$ & 13.8 & 12.5 & -1.3 \\
\hline Fucus serratus & $\mathrm{L}$ & - & - & - & - & - & - & 1.2 & 1.6 & 0.4 \\
\hline Halarachnion ligulatum & - & - & - & - & - & $\mathrm{L}$ & $\mathrm{L}$ & 7.0 & 12.5 & 5.5 \\
\hline Laminaria digitata & $\mathrm{L}$ & $\mathrm{L}$ & - & - & - & - & - & 1.9 & 2.5 & 0.6 \\
\hline Laminaria hyperborea & $\mathrm{L}$ & $\mathrm{L}$ & $\mathrm{L}$ & $\mathrm{L}$ & $\mathrm{L}$ & $\mathrm{L}$ & $L^{d}$ & 8.3 & $10.5^{\mathrm{e}}$ & $2.2^{\mathrm{e}}$ \\
\hline Lomentaria clavellosa & - & - & - & $\mathrm{L}$ & $\mathrm{L}$ & $\mathrm{L}$ & $\mathrm{L}$ & 7.0 & 12.5 & 5.5 \\
\hline Lomentaria orcadensis & - & - & - & $\mathrm{L}$ & L P & $\mathrm{L}$ & $\mathrm{L}$ & 8.3 & 12.5 & 4.2 \\
\hline Membranoptera alata & L P & L P & L P & L P & L P & - & - & 5.9 & 8.0 & 2.1 \\
\hline Phycodrys rubens & - & $\mathrm{L}$ & - & $\mathrm{L}$ & - & - & - & 7.5 & 6.0 & -1.5 \\
\hline Phyllophora pseudoceranoïdes & $\mathrm{L}$ & L P & $\mathrm{L}$ & $\mathrm{L}$ & $\mathrm{L}$ & - & - & 8.2 & 9.3 & 1.1 \\
\hline Plocamium cartilagineum & $\mathrm{L}$ & $\mathrm{L}$ & L P & L P & $\mathrm{L}$ & $\mathrm{L}$ & $\mathrm{L}$ & 9.0 & 11.5 & 2.5 \\
\hline Polyides rotundus & - & $\mathrm{L}$ & - & - & - & - & - & 3.2 & 1.9 & -1.3 \\
\hline Polysiphonia stricta & $\mathrm{P}$ & L P & L P & L P & L P & $\mathrm{L}$ & $\mathrm{L}$ & 9.6 & 12.5 & 2.9 \\
\hline Porphyropsis coccinea & - & - & $\mathrm{P}$ & - & $\mathrm{P}$ & - & - & 8.6 & 9.3 & 0.7 \\
\hline Pterothamnion plumula & - & - & - & $\mathrm{L}$ & $\mathrm{P}$ & $\mathrm{L}$ & - & 7.0 & 9.6 & 2.6 \\
\hline Rhodochorton purpureum & - & - & - & - & - & - & - & 8.0 & +0.4 & -8.4 \\
\hline Rhodomela confervoides & $\mathrm{L}$ & - & - & - & $\mathrm{L}$ & L & - & 7.3 & 10.2 & 2.9 \\
\hline Saccharina latissima & - & $\mathrm{P}$ & - & - & - & - & - & 2.9 & 2.0 & -0.9 \\
\hline Sargassum muticum & $\mathrm{L}$ & $\mathrm{L}$ & - & - & - & - & - & - & 2.4 & ${ }^{*}$ \\
\hline Ulva sp. & L P & L P & $\mathrm{P}$ & $\mathrm{P}$ & - & $\mathrm{L}$ & - & 9.0 & 9.6 & 0.6 \\
\hline Total number of species & 20 & 21 & 13 & 18 & 17 & 20 & 13 & & & \\
\hline
\end{tabular}

sent study. Another exception was Corallina officinalis, an easily identifiable conspicuous alga, which was only found $1.9 \mathrm{~m}$ shallower than in 1966-1968 (Lüning 1970). A few red algae (Ceramium virgatum, Chondrus crispus, Cystoclonium purpureum, Halarachnion ligulatum, Lomentaria clavellosa) and the brown alga Desmarestia aculeata occurred even 5.5 to $8.3 \mathrm{~m}$ deeper than in 1966-1968. The upper and lower limits of the dominant brown seaweeds Fucus serratus, Laminaria digitata and Saccharina latissima did not change, but adult L. hyperborea occurred $2.2 \mathrm{~m}$ deeper and $1 \mathrm{~m}$ shallower than in 1966-1968, thereby widening its zone of occurrence. Young thalli of the latter were found down to a depth of $12.5 \mathrm{~m}$.

\subsection{Biomass parameters of Laminaria spp. along the depth gradient}

Biomass, LAI and stipe length of Laminaria spp. along the depth gradient are presented in Tables 5 \& 6 . All biomass parameters of Laminaria plants were high between 0.5 and $4 \mathrm{~m}$ below MLWS and significantly decreased between 6 and $8 \mathrm{~m}$ below MLWS. In contrast, the biomass of epilithic, understory, noncrustose algae showed a drastic but insignificant increase below $4 \mathrm{~m}$ (Table 5), decreasing again at $10 \mathrm{~m}$ below MLWS (data not shown).

The LAI of Laminaria digitata and L. hyperborea showed the same trend as their biomass: high values of 
Table 5. Laminaria hyperborea. Biomass $\left(\mathrm{kg} \mathrm{m}^{-2}\right.$; total understory: $\mathrm{g} \mathrm{m}^{-2}$ ) along a depth gradient between 0.5 and $8 \mathrm{~m}$ below MLWS (mean of means $\pm \mathrm{SD}, \mathrm{n}=3$ ) and comparison of recent (2005; this study) and previous (Lüning 1969) data. 2005 means followed by different letters $(\mathrm{a}, \mathrm{b}, \mathrm{c}$ or combination of these) are significantly different $(\mathrm{p}<0.05)$. Fresh weight data from 1969 partially extracted from figures of Lüning (1969). Ldig: L. digitata; Lhyp: L. hyperborea; FW: fresh weight; DW: dry weight; nd: no data; (-) not included in statistical analyses. Total understory FW and mean blade DW: no significant differences (partially due to high SDs at 6 and $8 \mathrm{~m}$ )

\begin{tabular}{|c|c|c|c|c|c|c|c|}
\hline \multirow{4}{*}{ Biomass } & \multirow{4}{*}{$\begin{array}{l}\text { Year } \\
1969 \\
2005\end{array}$} & \multicolumn{6}{|c|}{ - Depth (m below MLWS) - } \\
\hline & & & 0.5 & 2 & 4 & $6^{\mathrm{a}}$ & 8 \\
\hline & & Ldig & nd & Lhyp & Lhyp & Lhyp & nd \\
\hline & & Ldig + Lhyp & Lhyp $^{b}$ & Lhyp & Lhyp & Lhyp & Lhyp \\
\hline \multirow[t]{3}{*}{ Whole plant FW } & 1969 & $5.6 \pm 0.9$ & nd & $11.1 \pm 1.8$ & $4.8 \pm 1.5$ & 0.1 & nd \\
\hline & 2005 & $6.6 \pm 0.3$ & $3.4 \pm 2.1$ & $6.4 \pm 2.4$ & $9.9 \pm 2.6$ & $3.2 \pm 0.8$ & $0.8 \pm 0.6$ \\
\hline & & $\mathrm{ac}$ & - & ac & a & bc & $\mathrm{b}$ \\
\hline \multirow[t]{2}{*}{ Whole plant DW } & 2005 & $1.6 \pm 0.3$ & $0.9 \pm 0.6$ & $1.8 \pm 0.4$ & $1.9 \pm 0.4$ & $0.7 \pm 0.2$ & $0.2 \pm 0.0$ \\
\hline & & a & - & a & a & $\mathrm{b}$ & $\mathrm{b}^{\mathrm{c}}$ \\
\hline \multirow[t]{3}{*}{ Blade FW } & 1969 & 4.7 & nd & 7.1 & nd & nd & nd \\
\hline & 2005 & $5.3 \pm 0.5$ & $2.5 \pm 1.5$ & $3.8 \pm 1.1$ & $5.7 \pm 1.8$ & $1.6 \pm 0.3$ & $0.6 \pm 0.5$ \\
\hline & & a & - & $\mathrm{ac}$ & $\mathrm{a}$ & bc & $\mathrm{b}$ \\
\hline \multirow[t]{2}{*}{ Single blade FW } & 1969 & nd & nd & $0.260 \pm 0.053$ & $0.292 \pm 0.091$ & nd & nd \\
\hline & 2005 & $0.138 \pm 0.047$ & $0.313 \pm 0.028$ & $0.232 \pm 0.028$ & $0.255 \pm 0.244$ & $0.117 \pm 0.025$ & $0.116 \pm 0.021$ \\
\hline \multirow[t]{3}{*}{ Blade DW } & 1969 & nd & nd & 1.4 & nd & nd & nd \\
\hline & 2005 & $1.3 \pm 0.2$ & $0.8 \pm 0.5$ & $1.2 \pm 0.3$ & $1.2 \pm 0.3$ & $0.4 \pm 0.1$ & $0.2 \pm 0.0$ \\
\hline & & $\mathrm{a}$ & - & $\mathrm{a}$ & a & b & b \\
\hline \multirow[t]{2}{*}{ Stipe FW } & 1969 & $0.9 \pm 0.2$ & nd & $4.0 \pm 1.3$ & $1.9 \pm 0.4$ & $<0.1$ & nd \\
\hline & 2005 & $\begin{array}{c}1.4 \pm 0.4 \\
\mathrm{bc}\end{array}$ & $\begin{array}{c}0.9 \pm 0.6 \\
-\end{array}$ & $\begin{array}{c}2.6 \pm 1.3 \\
\text { ac }\end{array}$ & $\begin{array}{c}4.1 \pm 1.0 \\
\text { a }\end{array}$ & $\begin{array}{c}1.6 \pm 0.5 \\
\mathrm{bc}\end{array}$ & $\begin{array}{c}0.3 \pm 0.2 \\
b\end{array}$ \\
\hline \multirow[t]{2}{*}{ Stipe DW } & 2005 & $0.3 \pm 0.1$ & $0.2 \pm 0.1$ & $0.6 \pm 0.2$ & $0.7 \pm 0.1$ & $0.3 \pm 0.1$ & $0.1 \pm 0.0$ \\
\hline & & bc & - & $\mathrm{ac}$ & $\mathrm{a}$ & bc & $\mathrm{b}$ \\
\hline Total understory FW & 2005 & $84.6 \pm 51.0$ & nd & $88.3 \pm 17.3$ & $223.0 \pm 85.0$ & $577.0 \pm 283.0$ & $906.9 \pm 544.6$ \\
\hline
\end{tabular}

Table 6. Laminaria hyperborea. Population density (ind. $\mathrm{m}^{-2}$ ) and morphometric parameters along a depth gradient between 0.5 and $8 \mathrm{~m}$ below MLWS (mean of means $\pm \mathrm{SD}, \mathrm{n}=3$ ). Comparison of recent (2005; this study) and previous (Lüning 1969) data. Data from 1969 partially extracted from figures of Lüning (1969). 2005 means followed by different letters (a, b, c, or combination of these) are significantly different ( $\mathrm{p}<0.05)$. Ldig: L. digitata, Lhyp: L. hyperborea, nd: no data. Stipe length: no significant differences due to high SDs at $2 \mathrm{~m}$

\begin{tabular}{|c|c|c|c|c|c|c|c|c|}
\hline \multirow[t]{2}{*}{ Biomass } & \multirow[t]{2}{*}{ Year } & \multicolumn{7}{|c|}{ - Depth (m below MLWS) } \\
\hline & & 0.5 & 0.5 & 2 & 3 & 4 & 6 & 8 \\
\hline & $\begin{array}{l}1969 \\
2005\end{array}$ & $\begin{array}{c}\text { Ldig } \\
\text { Ldig + Lhyp }\end{array}$ & $\stackrel{-}{\text { Lhyp }^{a}}$ & $\begin{array}{l}\text { Lhyp } \\
\text { Lhyp }\end{array}$ & $\begin{array}{l}\text { Lhyp } \\
\text { nd }\end{array}$ & $\begin{array}{l}\text { Lhyp } \\
\text { Lhyp }\end{array}$ & $\begin{array}{l}\text { Lhyp } \\
\text { Lhyp }\end{array}$ & $\begin{array}{l}\text { nd } \\
\text { Lhyp }\end{array}$ \\
\hline Density (ind. $>20 \mathrm{~cm}$ ) & 1969 & nd & nd & $27 \pm 5.7$ & $13 \pm 6.7$ & $10 \pm 1.4$ & 0.9 & nd \\
\hline $\begin{array}{l}\text { Density (ind. stipe } \\
\text { length }>10 \mathrm{~cm}^{\mathrm{b}} \text { ) }\end{array}$ & 2005 & $\begin{array}{c}25.7 \pm 6.4 \\
\text { ab }\end{array}$ & $\begin{aligned} 6.3 & \pm 7.5 \\
& -\end{aligned}$ & $\begin{array}{c}31.0 \pm 21.1 \\
a b\end{array}$ & $\begin{array}{l}\text { nd } \\
\text { nd }\end{array}$ & $\begin{array}{c}35.3 \pm 7.2 \\
\mathrm{a}\end{array}$ & $\begin{array}{c}15.3 \pm 6.0 \\
a b\end{array}$ & $\begin{array}{c}3.7 \pm 4.0 \\
\mathrm{~b}\end{array}$ \\
\hline Leaf area index & $\begin{array}{l}1969 \\
2005\end{array}$ & $\begin{array}{c}4.7 \pm 0.8 \\
5.3 \pm 0.9 \\
a\end{array}$ & $\begin{array}{c}\text { nd } \\
2.6 \pm 1.5 \\
-\end{array}$ & $\begin{array}{c}4.1 \pm 0.3 \\
3.3 \pm 1.1 \\
\text { ac }\end{array}$ & $\begin{array}{l}2.3 \\
\text { nd } \\
\text { nd }\end{array}$ & $\begin{array}{c}1.6 \pm 0.5 \\
4.4 \pm 0.9 \\
\mathrm{a}\end{array}$ & $\begin{array}{c}\text { nd } \\
1.3 \pm 0.5 \\
\text { bc }\end{array}$ & $\begin{array}{c}\text { nd } \\
0.3 \pm 0.3 \\
b\end{array}$ \\
\hline Stipe length $(\mathrm{cm})$ & 2005 & $31.6 \pm 4.9$ & $46.1 \pm 6.0$ & $54.5 \pm 52.1$ & nd & $33.6 \pm 0.8$ & $29.9 \pm 10.8$ & $16.4 \pm 12.7$ \\
\hline
\end{tabular}


3.3 to 5.5 occurred between 0.5 and $4 \mathrm{~m}$ below MLWS and significantly lower values of 1.3 and 0.3 were encountered at 6 and $8 \mathrm{~m}$ below MLWS, respectively (Table 6).

Maximum stipe lengths of up to $123 \mathrm{~cm}$ were present at $2 \mathrm{~m}$ below MLWS and then decreased with increasing depth (not shown). However, differences in mean stipe lengths at different depths were not significant (Table 6), although maximum stipe lengths indicated such a trend. In contrast, stipe biomass of Laminaria hyperborea was significantly higher at $4 \mathrm{~m}$ below MLWS than at 0.5, 6 and $8 \mathrm{~m}$ below MLWS (Table 5).

The mean population density of Laminaria hyperborea with stipe lengths $\geq 10 \mathrm{~cm}$ showed an insignificant increase from 25.7 ind. $\mathrm{m}^{-2}$ at $0.5 \mathrm{~m}$ below MLWS (here together with L. digitata) to 31.0 ind. $\mathrm{m}^{-2}$ at $2 \mathrm{~m}$ below MLWS and a maximum of 35.3 ind. $\mathrm{m}^{-2}$ at $4 \mathrm{~m}$ below MLWS. Below this depth, the mean density decreased to 15.3 ind. $\mathrm{m}^{-2}$ at $6 \mathrm{~m}$ and significantly to 3.7 ind. $\mathrm{m}^{-2}$ at $8 \mathrm{~m}$ below MLWS (Table 6). Although no L. hyperborea was found in the random quadrats at $10 \mathrm{~m}$ depth, they were observed at this depth.

The LAI, density and biomass $\left(\mathrm{kg} \mathrm{FW} \mathrm{m}^{-2}\right)$ of Laminaria species differed between 1969 and 2005 (Tables $5 \& 6$; Fig. 3). LAI at $4 \mathrm{~m}$ and density at 4 and $6 \mathrm{~m}$ below MLWS were significantly higher in 2005 than in 1969, whereas no significant differences were obvious at 0.5 and $2 \mathrm{~m}$ below MLWS. Similarly, biomass significantly increased at 0.5, 4 and $6 \mathrm{~m}$ below MLWS in 2005 compared to 1969, but significantly decreased at $2 \mathrm{~m}$ below MLWS.

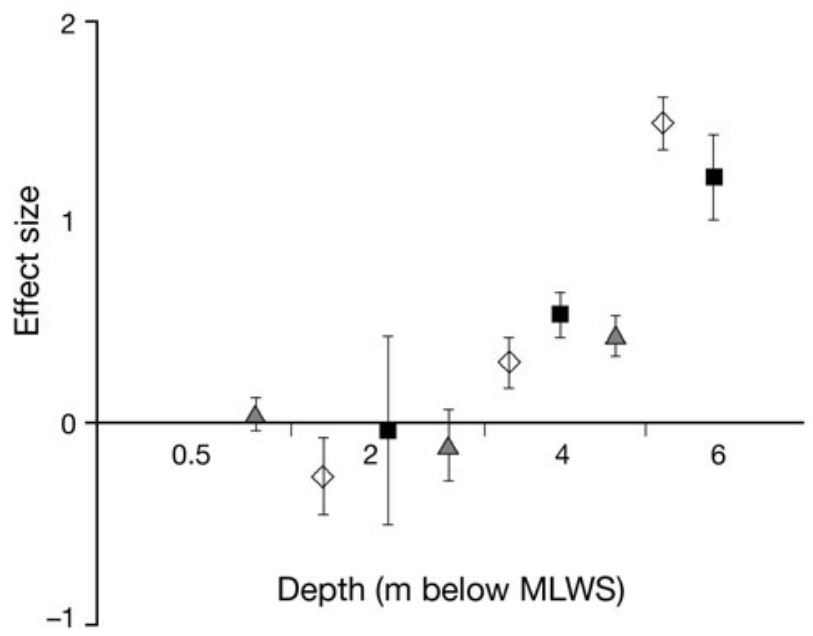

Fig. 3. Laminaria hyperborea. Effect size of ( $\Delta$ ) LAI (leaf area index), ( $\square$ ) density of individuals (ind. $\mathrm{m}^{-2}$ ) and $(\diamond)$ biomass $\left(\mathrm{kg} \mathrm{FW} \mathrm{m^{-2 }}\right.$ ) in relation to depth below MLWS (mean $\pm 95 \%$ $\mathrm{CI}, \mathrm{n}=3$ ). Positive values indicate an increase, negative values a decrease, of the measured parameter compared to those from Lüning (1969). Significance is indicated when whiskers of the CI do not cross the $x$-axis

\section{DISCUSSION}

The data presented reveal that the depth distribution of seaweeds and the biomass and structure of the kelp beds off Helgoland have significantly changed between 1966-1968 and 2005-2006. The most important changes are (1) 2 to $8 \mathrm{~m}$ increase in the lower depth limits in $50 \%$ of the recorded noncrustose algal species, (2) a shift in the biomass maximum of Laminaria hyperborea from 2 to $4 \mathrm{~m}$ below MLWS, (3) significant increases in biomass, leaf area index and density of $L$. hyperborea at 0.5, 4 and $6 \mathrm{~m}$ below MLWS and extension of the dense L. hyperborea forest (LAI >1) towards shallower and deeper areas; and (4) a change in the community structure of the kelp bed evident in the drastic decrease of the relative presence and abundance of the sugar kelp Saccharina latissima in the northern transect T1, the increase in its depth distribution in Transect T2, the widening of the L. hyperborea zone and invasion of the Sargasso weed Sargassum muticum.

\subsection{Change in lower depth limits}

The described $\sim 2$ to $8 \mathrm{~m}$ increase in the depth distribution ranges of sublittoral seaweed species around the island of Helgoland appears to reflect the increased water transparency reported for Helgoland. Mean yearly Secchi depths increased by $1 \mathrm{~m}$ between 1975 and 2001 (Wiltshire et al. 2008), increasing steadily over time. However, given that low yearly mean Secchi depths $(<3 \mathrm{~m})$ were recorded only prior to 1984, there might have been a more rapid increase in water transparency since then. Although Secchi depths have been recorded every weekday since 1975, these have not yet been analysed by season, thus precluding more detailed analysis. The situation at Helgoland runs parallel to the general situation in the North Sea which has become less turbid in recent years (McQuatters-Gallop et al. 2007). Generally, the depth range of seaweeds corresponds to irradiance levels which are a function of water depth and Jerlov water types. At Helgoland, the water body is dominated by coastal Jerlov Water Type 7 (irradiance transmittance $<65 \%$, maximal transmittance in green light between 480 and $580 \mathrm{~nm}$; Lüning 1981) during the main growth period from April to September. The total annual light received at the lower kelp limit corresponds to $0.7 \%$ of the surface irradiance at Helgoland and is generally set at $\sim 1 \%$ of the surface irradiance for kelps and at 0.05 to $0.1 \%$ for other multicellular algae (Lüning \& Dring 1979). The relationship between Secchi depths and underwater irradiance availability has been determined only once at Helgoland (Dring \& 
Lüning 1994). Two points have to be taken into account here: (1) Under conditions of identical light attenuation in the water column, the same Secchi depth may reflect different underwater irradiance regimes in summer than in winter as (2) there is considerable variability due to seasonal variation in incident light. This may result in Secchi depths with opposing trends between seasons, as has been observed in the southern North Sea (Cadée \& Hegeman 2002). This certainly is also the case for Helgoland where underwater light is strongly reduced during winter months (Lüning \& Dring 1979). However, correlation between seasonal Secchi depth changes and active growth periods of seaweeds is still missing. As turbidity is not only connected to eutrophication but also to wind stress and irradiance changes, the proportion of de-eutrophication and climate change effects that ameliorate underwater irradiance at Helgoland is yet unclear.

A decrease in depth limits has been variously reported (e.g. Pedersen \& Snoeijs 2001) to be an indirect consequence of eutrophication that has degraded the light climate. However, depth limits for Fucus vesiculosus at some selected Baltic sites have recently increased (Torn et al. 2006). Secchi depths also explained most of the variation in the depth limits, but the observed trends in all investigated districts did not clearly follow reported changes in nutrient or light levels and are thus not attributable to de-eutrophication alone (Torn et al. 2006). Five red and one brown algal species at Helgoland shifted their maximum depths of occurrence by 5.5 to $8.3 \mathrm{~m}$ which exceeds the reported Secchi depth increase of $1 \mathrm{~m}$ as well as the methodological differences (number of transects, accuracy of depth gauge of both investigations) which could only account for $\sim 1 \mathrm{~m}$. Thus, the depth changes at Helgoland do not merely follow mean Secchi depths. Differences between species in algal depth changes have also been observed elsewhere (Pedersen \& Snoeijs 2001). Species-specific seasonal growth patterns and different low-light acclimation potentials (e.g. Wiencke 1990) may interact in a still unknown manner with seasonal and long-term light availability.

A variety of species that were present between 10 and $13.5 \mathrm{~m}$ below MLWS in 2005 were not recorded by Lüning (1970) at these depths. Previously, crustose species and the small red algae Erythrodermis traillii and Colaconema membranaceum dominated at $>10 \mathrm{~m}$ below MLWS (Lüning 1970), while another 11 algal species were recorded at this level in the present study. These include Bonnemaisonia hamifera (Trailliella-phase), Brongniartella byssoides, Delesseria sanguinea, Halarachnion ligulatum, Lomentaria clavellosa and L. orcadenis, most of them typical deep water species. The depth limit for crustose species had been 15 m below MLWS in 1966-1968 (Lüning 1970) with occasional records at $21 \mathrm{~m}$ below MLWS (K. Lüning pers. comm.). This corresponds to observations of de Kluijver (1997) who reported crustose algae at $23 \mathrm{~m}$ below MLWS. In 2005 and 2006, this limit was not investigated.

In a few cases, obvious changes of upper depth limits were also observed. Most prominent is that of the deep red alga Halarachnion ligulatum. This species was only recorded between 9.6 and $12.5 \mathrm{~m}$ below MLWS in 2005 (Table 4), while it was restricted to 4.5 to $8.5 \mathrm{~m}$ below MLWS in 1966-1968 (Lüning 1970). This could either be a sampling artefact due to the reduced number of investigated quadrats in 2005 or may indicate susceptibility to higher irradiance. Pang et al. (2001) reported that UV radiation may limit the upper distribution of the deepwater species Delesseria sanguinea.

\subsection{Change in the structure of the kelp bed}

For comparative purposes, Lüning's concept of vegetation zones was adopted. Although it is generally accepted that there is zonation of dominant brown algae along the depth gradient in the sublittoral similar to the zonation of Fucales in the intertidal (Lüning 1990), there is some debate whether these zones represent distinct floristic communities or a continuum (see discussion in Kent \& Coker 1992). The similarity analysis of quantitative percentage cover values of quadrats taken along the depth gradient and pre-classified to vegetation zones (see Table 2) revealed that these zones can only be partially separated (Fig. 4). Species composition and abundance in the mixed Fucus serratus/Laminaria digitata zone, the pure L. digitata zone and the mixed L. digitata/L. hyperborea zone are indistinguishable. The pure F. serratus zone is similar to this cluster, but separated. The pure L. hyperborea zone is clearly distinct from all other zones, while the $L$. hyperborea-park vegetation and red algal zone form a cline following the depth of occurrence. Thus, while these vegetation zones are only partially discrete entities, the widths and depths of occurrence of the dominant brown seaweeds nevertheless provide a good indication of stability or change within the kelp bed.

Although the number of investigated depths and $5 \mathrm{~m}$ intervals differed between the present study and that of Lüning (2005-2006: 2 transects; 6075 m intervals, $0.9 \mathrm{~m}$ above to $13.5 \mathrm{~m}$ below MLWS vs. 1966-1968: 5 transects, $5375 \mathrm{~m}$ intervals, 0.2 to $8.3 \mathrm{~m}$ below MLWS), major changes in zonation patterns and depth distribution were detected: the pure vegetation of Fucus serratus and Laminaria digitata was shifted into shallower areas around $1 \mathrm{~m}$ below MLWS in 2005. Furthermore, pure Saccharina latissima stands were no 


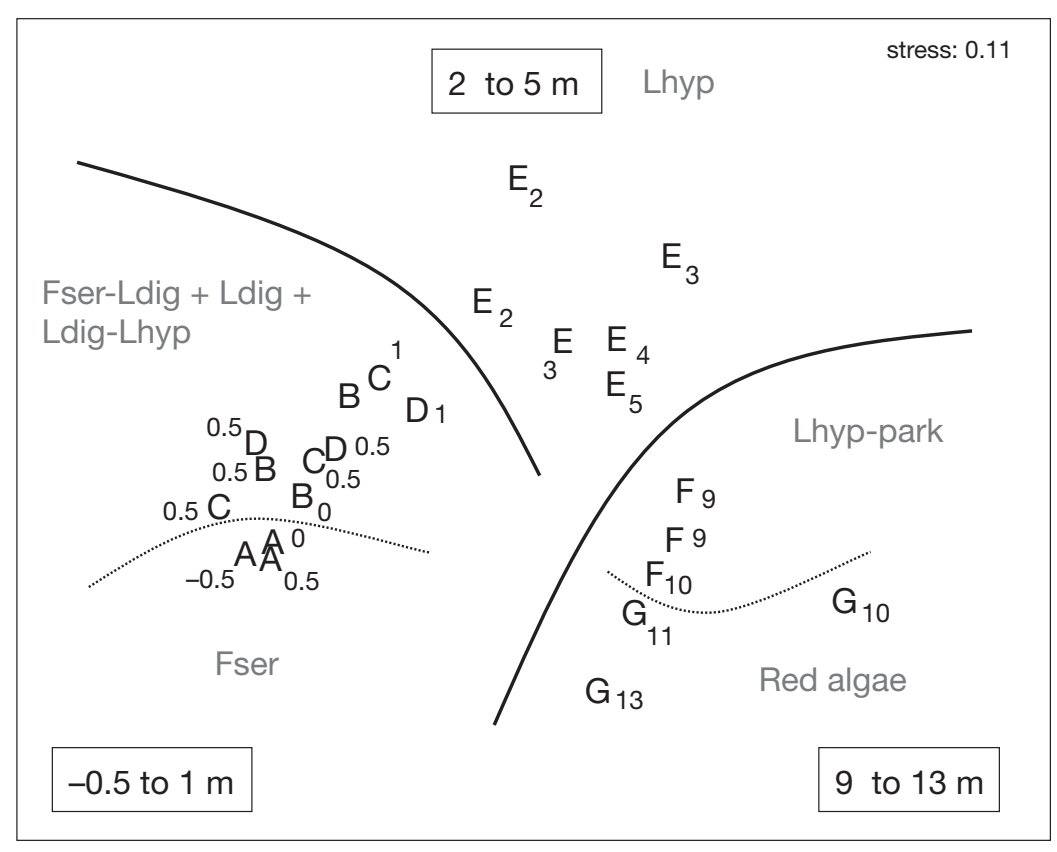

Fig. 4. Results of n-MDS analyses of quantitative percentage cover data (square root transformed) along Transect T1 with zone and depth classification. Letters refer to the predefined brown algal zones (see Table 2; also for abbreviations) and numbers beside letters indicate depth below MLWS

longer present in T1 in 2005, and had shifted to greater depths in T2 (in 2006). In 1966-1968, L. digitata and $S$. latissima together were the most important species of the upper sublittoral (0.4 to $1.3 \mathrm{~m}$ below MLWS), while presently this zone is mostly occupied by L. digitata alone. The mixed $L$. digitata/L. hyperborea vegetation did not exist previously and the pure L. hyperborea forest extended both shallower (at $1.3 \mathrm{~m}$ below MLWS in 2005 vs. 2.5 m below MLWS in Fig. 7 of Lüning 1970) and deeper (park vegetation at $10.5 \mathrm{~m}$ below MLWS in 2005 vs. 8 to $9 \mathrm{~m}$ below MLWS in 1966-1968). These distributional changes and the changes in biomass, density and LAI indicate that the L. hyperborea forest extended its range upwards and downwards; upwards most probably at the expense of $S$. latissima and L. digitata stands. Although pure $S$. latissima stands still occur at T2 north of Dune Island (Fig. 2), vegetation structure also changed here. Today, most pure S. latissima stands occur between 3.5 and $5.5 \mathrm{~m}$ below MLWS and therefore much deeper than reported in 19661968 (1.1 to 2 m below MLWS; Fig. 7 in Lüning 1970). Furthermore, mixed S. latissima/L. hyperborea stands which were not present in the late 1960s (Lüning 1970) presently occur between 0.5 and $5.5 \mathrm{~m}$ below MLWS. Old reports from Helgoland mentioned $4 \mathrm{~m}$ long thalli of S. latissima (Hallier 1863, Kuckuck 1897, Nienburg 1925, Lüning 1970) and noted the ubiquity of this species, which formed a closed girdle in the infralittoral zone around the entire island (Hallier 1863, Kuckuck
1894, Nienburg 1925). This situation has completely changed. At least since 1999 (I. Bartsch pers. obs.), no infralittoral fringe of $S$. latissima has been reported from the northern part of the island; mostly small and young individuals were found along T1 during the present investigation, but adult plants were still present in low quantities along T2. Similar drastic population losses of $S$. latissima have been reported from Norway (Moy et al. 2003) and France (Givernaud et al. 1991, Cosson 1999), for reasons that are still unknown.

The increased water clarity and therefore increased depth penetration of UV radiation and photosynthetically active radiation (PAR) may have affected the kelp population at Helgoland. Kelp zoospore photosynthesis is adapted to low irradiation conditions and in Helgoland, Laminaria hyperborea and Saccharina latissima zoospores are clearly more sensitive to higher photon flux rates than $L$. digitata zoospores (Roleda et al. 2005). Young S. latissima sporophytes are more susceptible to UV radiation than those of L. hyperborea and L. digitata (Dring et al. 1996), but germination of zoospores of Helgolandic $S$. latissima and $L$. digitata have not been affected by UVA (Roleda et al. 2005). Arctic S. latissima zoospores are also more sensitive to UV radiation compared to 3 other Arctic kelp species (Roleda et al. 2006). These findings may partially explain the presently deeper distribution of $S$. latissima at Helgoland but does not explain the extension of $L$. hyperborea towards the water surface. Thus, the structural changes cannot be accounted for by improvement of water clarity alone. Another factor acting in the shallow sublittoral is wave exposure which is dependent on wind stress. S. latissima prefers more wave protected sites compared to $L$. digitata and L. hyperborea (Kain 1962). The increase in storm activity and extreme wave height over the southern North Sea between 1958 and 2001 (e.g. Weisse et al. 2005) may have affected the kelp population at Helgoland.

Finally, temperature increases affect the distribution of kelp species (Breeman 1988). Mean summer surface water temperatures at Helgoland were reported to be $\sim 18^{\circ} \mathrm{C}$ (Lüning 1990) with maxima at $20^{\circ} \mathrm{C}$ but have been higher in recent years (K. H. Wiltshire unpubl. data); mean summer surface temperature has increased by $1.33^{\circ} \mathrm{C}$ in the last $40 \mathrm{yr}$ (Wiltshire et al. 2008). Peak summer surface temperatures are close to 
the survival limit of Helgolandic Laminaria hyperborea, L. digitata and Saccharina latissima (Fortes \& Lüning 1980, tom Dieck 1992). Uppermost L. digitata in Helgoland (I. Bartsch pers. obs.) and L. hyperborea in Northern Spain (J. Rico pers. comm.) may suffer after hot summers, and spore germination of L. digitata is hampered even during 'normal' summers (J. S. Vogt \& I. Bartsch pers. comm.). Evidence of negative effects of hot summers on the life cycle of $S$. latissima is yet missing. Nevertheless, migration of kelp species into deeper and somewhat cooler water is probable if water temperatures increase.

\subsection{Change in biomass, LAI and population density}

The biomass of Laminaria in general (overview in Bartsch et al. 2008) and specifically of L. hyperborea have been previously investigated (e.g. Lüning 1969, Gunnarsson 1991, Sjøtun et al. 2004). As biomass values are dependent on density of stands, season and latitude, the numbers available in the literature are difficult to compare. The biomasses of L. hyperborea and $L$. digitata stands vary between 5 to $40 \mathrm{~kg} \mathrm{FW} \mathrm{m}^{-2}$ (see Lüning 1969, Gunnarsson 1991, Sjøtun et al. 2004). With a maximum of $9.9 \pm 2.6 \mathrm{~kg}$ FW L. hyperborea $\mathrm{m}^{-2}$ recorded within a dense undisturbed stand in August (the time period of its maximum blade size, Lüning 1979) (Table 5), Helgoland may thus be regarded as a mean to low production site.

Comparison of present and previous biomass, LAIand population density data from Helgoland confirm the trend in depth shift described above for the algal depth limits. Although the kelp biomass remained nearly constant at 0.5 and $2 \mathrm{~m}$ below MLWS, the species composition changed: in 1966-1968, Saccharina latissima was present at this depth, but it is partially replaced by Laminaria hyperborea today. The presentday $55 \%$ biomass increase from 2 to 4 below MLWS contrasts with a $57 \%$ biomass decrease from 2 to $4 \mathrm{~m}$ below MLWS in 1966-1968. This may be explained by natural variability of population density and the sample size in this study. Two of the 3 quadrats at $2 \mathrm{~m}$ below MLWS showed relatively low biomass but high density values, being composed of relatively young specimens $(<1$ to $3 \mathrm{yr}$, only $9-27 \%$ of individuals older than 3 yr), while one quadrat showed the opposite (few old and heavy plants, 6 to $7 \mathrm{yr}$ old; data not shown) and thus had comparable data as that of Lüning (1969). Due to this age variance of the algae in the investigated quadrats at $2 \mathrm{~m}$ below MLWS, the SD of all investigated parameters at this depth was very high, resulting in insignificant differences, although clear trends were visible. Comparing the SDs at 2 and $4 \mathrm{~m}$ below MLWS, it is also apparent that the Laminaria forest is more stable at $4 \mathrm{~m}$ than at $2 \mathrm{~m}$ below MLWS. Hence, the extent of sampling should be increased in future studies. Despite the depth shift of $2 \mathrm{~m}$, maximum biomass remained rather constant over time, as 2005 biomass data at 4, 6 and $8 \mathrm{~m}$ below MLWS corresponded to values at 2, 4 and $6 \mathrm{~m}$ below MLWS in 1969. However, total kelp biomass increased due to the expansion of the L. hyperborea forest.

In conclusion, this study shows that significant changes have occurred in the depth limits, community structure, and biomass of dominant brown seaweeds at Helgoland between 1966-1968 and 2005-2006. Most of these changes are probably attributable to the observed increase in water clarity, but changes in temperature and storminess may also have had an effect. As the reported increase in water transparency is accompanied by a decrease in phosphate concentration of $40 \%$ (Wiltshire et al. 2008), the observed changes in depth distribution and biomass of seaweeds may also reflect de-eutrophication. However, Wiltshire et al. (2008) assume that this situation reflects a hydrographic shift to more marine conditions at Helgoland correlated with increased southwesterly wind events. The same paper cites unpublished data by Stockmann and colleagues who showed a shift to a less coastally dominated system for Helgoland. De-eutrophication is normally correlated with a decrease in primary production and biomass (e.g. Cadée \& Hegeman 2002) which has not been observed here and in phytoplankton systems in the North Sea (McQuatters-Gallop et al. 2007, Wiltshire et al. 2008). The probability of an altered circulation pattern with more inflow of clear Atlantic water during a positive phase of the North Atlantic Oscillation has been discussed in several papers (e.g. Ottersen et al. 2001, Edwards et al. 2002, McQuatters-Gallop et al. 2007). This and the observed changes in water clarity should be taken into consideration for the southern North Sea and Helgoland.

Acknowledgements. C.P. and I.B. thank the diving crew of the Biologische Anstalt Helgoland, especially C. Wanke and S. Brandt, for their invaluable help and constant support; the State Office for Nature and Environment (LANU), Schleswig-Holstein for partial financial support; P. Schubert and T. Kaschell for help with the field work; M. Molis for support in statistics and data evaluation; H. Pehlke and J. Bartsch for help with GIS topics; T. Schütte for collecting data for Transect T2; and J. Bartsch for correction of the manuscript. We also thank $\mathrm{K}$. Lüning for providing us with his original material. This work was part of the M.Sc. thesis of C.P. at the University of Bremen, Germany. Publication content complies with German laws.

\section{LITERATURE CITED}

Bartsch I, Kuhlenkamp R (2000) The marine macroalgae of Helgoland (North Sea): an annotated list of records between 1845 and 1999. Helgol Mar Res 54:160-189 
Bartsch I, Tittley I (2004) The rocky intertidal biotopes of Helgoland: present and past. Helgol Mar Res 58:289-302

Bartsch I, Wiencke C, Bischof K, Buchholz CM and 14 others (2008) The genus Laminaria sensu lato: recent insights and developments. Eur J Phycol 43:1-86

Braun-Blanquet J (1951) Pflanzensoziologie: Grundzüge der Vegetationskunde. Springer, Wien

Breeman AM (1988) Relative importance of temperature and other factors in determining geographic boundaries of seaweeds: experimental and phenological evidence. Helgol Mar Res 42:199-241

> Cadée GC, Hegeman J (2002) Phytoplankton in the Marsdiep at the end of the 20th century; 30 years monitoring biomass, primary production, and Phaeocystis blooms. J Sea Res 48:97-110

> Cosson J (1999) Sur la disparition progressive de Laminaria digitata sur les côtes du Calvados (France). Cryptogam Algol 20:35-42

de Kluijver MJ (1997) Sublittoral communities of the North Sea hard-substrata. $\mathrm{PhD}$ thesis, University of Amsterdam

den Hartog C (1959) The epilithic algal communities occurring along the coast of the Netherlands. Wentia 1:1-241

> Dring MJ, Lüning K (1994) Influence of spring-neap tidal cycles on the light available for photosynthesis by benthic marine plants. Mar Ecol Prog Ser 104:131-137

Dring MJ, Wagner A, Boskov J, Lüning K (1996) Sensitivity of intertidal and subtidal red algae to UVA and UVB radiation, as monitored by chlorophyll fluorescence measurements, influence of collection depth and season, and length of irradiation. Eur J Phycol 31:293-302

Dubi A, Tørum A (1995) Wave damping by kelp vegetation. In: Edge BL (ed) Proc 24th Int Conf on Coastal Engineering, Kobe, Japan, 22-28 Oct., 1994, American Society of Civil Engineers, p 142-156

Edwards M, Beaugrand G, Reid PC, Rowden AA, Jones MB (2002) Ocean climate anomalies and the ecology of the North Sea. Mar Ecol Prog Ser 239:1-10

Fortes MD, Lüning K (1980) Growth rates of North Sea macroalgae in relation to temperature, irradiance and photoperiod. Helgol Mar Res 34:15-29

Franke HD, Gutow L (2004) Long-term changes in the macrozoobenthos around the rocky island of Helgoland (German Bight, North Sea). Helgol Mar Res 58:303-310

Givernaud T, Cosson J, Givernaud-Mouradi A (1991) Étude des populations de Sargassum muticum (Yendo) Fensholt sur les côtes de Basse-Normandie (France). In: Elliott M, Ducrotony JP (eds) Estuaries and coasts: spatial and temporal intercomparisons. 19th ECSA Symp, Olsen \& Olsen, Fredensborg, p 129-132

Greig-Smith P (1983) Quantitative plant ecology. Studies in ecology, Vol 9, 3rd edn. Blackwell, Oxford

Gunnarsson K (1991) Populations de Laminaria hyperborea et Laminaria digitata (Phéophycées) dans la Baie de Breidifjördur, Islande. J Mar Institue Reykjavik 12:1-148

Hallier E (1863) Vegetation auf Helgoland. Otto Meißner, Hamburg

Kain JM (1962) Aspects of the biology of Laminaria hyperborea, I. Vertical distribution. J Mar Biol Assoc UK 42: 377-385

Kautsky N, Kautsky H, Kautsky U, Waern M (1986) Decreased depth penetration of Fucus vesiculosus (L.) since the 1940's indicates eutrophication of the Baltic Sea. Mar Ecol Prog Ser 28:1-8

Kent M, Coker P (1992) Vegetation description and analysis. A practical approach. Belhaven Press, London

Kuckuck P (1894) Bemerkungen zur marinen Algenvegetation von Helgoland. Wiss Meeresunter 1:223-263
Kuckuck P (1897) Ueber marine Vegetationsbilder. Ber Dtsch Bot Ges 15:441-447

Lüning K (1969) Standing crop and leaf area index of the sublittoral Laminaria species near Helgoland. Int J Life Oceans Coast Waters 3:282-286

Lüning K (1970) Tauchuntersuchungen zur Vertikalverteilung der sublitoralen Helgoländer Algenvegetation. Helgol wiss Meeresunters 21:271-291

Lüning K (1979) Growth strategies of three Laminaria species (Phaeophyceae) inhabiting different depth zones in the sublittoral region of Helgoland (North Sea). Mar Ecol Prog Ser 1:195-207

Lüning K (1981) Light. In: Lobban CS, Wynne MJ (eds) The biology of seaweeds. Blackwell, Oxford, p 326-355

Lüning K (1990) Seaweeds. Their environment, biogeography and ecophysiology. Wiley, New York

Lüning K, Dring MJ (1979) Continuous underwater light measurement near Helgoland (North Sea) and its significance for characteristic light limits in the sublittoral region. Helgol Wiss Meeresunters 32:403-424

- Markham JW, Munda IM (1980) Algal recolonization in the rocky eulittoral at Helgoland, Germany. Aquat Bot 9:33-71

McQuatters-Gallop A, Raitsos DE, Edwards M, Pradhan Y, Mee LD, Lavender SJ, Attrill MJ (2007) A long-term chlorophyll data set reveals regime shift in North Sea phytoplankton biomass unconnected to nutrient trends. Limnol Oceanogr 52:635-648

Moy F, Aure J, Dahl E, Green N and 9 others (2003) Landtidsovervåking av miljøkvaliteten i kystområdene av Norge. Årsrapport for 2002, p 1-69

Munda IM, Markham JW (1982) Seasonal variations of vegetation patterns and biomass constituents in the rocky eulittoral of Helgoland. Helgol Mar Res 35:131-151

Nienburg W (1930) Die Besiedelung des Felsstrandes und der Klippen von Helgoland, Teil II. Die Algen. Helgol Wiss Meeresunters 15:1-15

- Ottersen G, Planque B, Belgrano A, Post E, Reid PC, Stenseth NC (2001) Ecological effects of the North Atlantic oscillation. Oecologia 128:1-14

Pang S, Gomez I, Lüning K (2001) The red macroalga Delesseria sanguinea as a UVB-sensitive model organism: selective growth reduction by UVB in outdoor experiments and rapid recording of growth rate during and after UV pulses. Eur J Phycol 36:207-216

Pedersen M, Snoeijs P (2001) Patterns of macroalgal diversity, community composition and long-term changes along the Swedish west coast. Hydrobiologia 459:83-102

> Reichert K, Buchholz F (2006) Changes in the macrozoobenthos of the intertidal zone at Helgoland (German Bight, North Sea): a survey of 1984 repeated in 2002. Helgol Mar Res 60:213-223

Reichert K, Buchholz F, Bartsch I, Kersten T, Giménez L (2008) Scale-dependent patterns of variability in species assemblages of the rocky intertidal at Helgoland (German Bight, North Sea). J Mar Biol Assoc UK (in press)

Roleda MY, Wiencke C, Hanelt D, van de Poll WH, Gruber A (2005) Sensitivity of Laminariales zoospores from Helgoland (North Sea) to ultraviolet and photosynthetically active radiation: implications for depth distribution and seasonal reproduction. Plant Cell Environ 28:466-479

Roleda MY, Hanelt D, Wiencke C (2006) Exposure to ultraviolet radiation delays photosynthetic recovery in Arctic kelp zoospores. Photosynth Res 88:311-322

Schultze K, Janke K, Krüß A, Weidemann W (1990) The macrofauna and macroflora associated with Laminaria digitata and Laminaria hyperborea at the island of Helgoland (German Bight, North Sea). Helgol Mar Res 44:39-51 
Sjøtun K, Olsen BR, Eggereide SF (2004) Biomassekartlegging av Laminaria digitata i Smøla-området - Biomass survey of Laminaria digitata in the Smøla area. Fisken og havet 11, p 20

tom Dieck (Bartsch) I (1992) North Pacific and North Atlantic digitate Laminaria species (Phaeophyta): hybridization experiments and temperature responses. Phycologia 31: $147-163$

Torn K, Krause-Jensen D, Martin G (2006) Present and past distribution of bladderwrack (Fucus vesiculosus) in the Baltic. Aquat Bot 84:53-62

Underwood JA (1997) Experiments in ecology: their logical design and interpretation using analysis of variance. Cambridge University Press, Cambridge

Submitted: October 18, 2007; Accepted: July 30, 2008
Weisse R, von Storch H, Feser F (2005) Northeast Atlantic and North Sea storminess as simulated by a regional climate model 1958-2001 and comparison with observations. J Clim 18:465-479

Wiencke C (1990) Seasonality of red and green macroalgae from Antarctica - a long-term culture study under fluctuating Antarctic daylength. Polar Biol 10:601-607

Wiltshire KH, Manly BFJ (2004) The warming trend at Helgoland Roads, North Sea: phytoplankton response. Helgol Mar Res 58:269-273

Wiltshire $\mathrm{KH}$, Malzahn AM, Wirtz K, Greve W and others (2008) Resilience of North Sea phytoplankton spring bloom dynamics: an analysis of long-term data at Helgoland Roads. Limnol Oceanogr 53:1294-1302

Proofs received from author(s): September 19, 2008 